\title{
Uncommon Ethiology in Abdominal Pain: Celiac Axis Compression Syndrome
}

\author{
Serife Degirmencioglu1, Banu Boyuk1, Hande Atalay², Muhammed Ates1, Murat Altay', \\ Aslan Celebi1 ${ }^{1}$, Ismail Ekizoglu ${ }^{1}$ \\ ${ }^{1}$ Department of Internal Medicine, Gaziosmanpasa Taksim Education and Research Hospital, Istanbul, Turkey \\ ${ }^{2}$ Department of Internal Medicine, Gebze Fatih Government Hospital, Kocaeli, Turkey \\ Email: handeerman@yahoo.com
}

Received 9 December 2015; accepted 11 January 2016; published 15 January 2016

Copyright (C) 2016 by authors and Scientific Research Publishing Inc.

This work is licensed under the Creative Commons Attribution International License (CC BY). http://creativecommons.org/licenses/by/4.0/

c) (i) Open Access

\begin{abstract}
Celiac axis compression syndrome, one of the reasons of mesenteric ischemia, is an extremely rare etiology of abdominal pain. Primary pathological mechanism is the external compression of the celiac trunk by median arcuate ligament. The diagnosis of this condition is usually difficult and depends on angiographic findings and computerized tomography (CT) evaluations. Here we report a celiac axis compression syndrome case, presenting with chronic abdominal pain and weight loss, which was successfully treated by surgery.
\end{abstract}

\section{Keywords}

Abdominal Pain, Mesenteric Ischemia, Median Arcuate Ligament, Celiac Artery Compression

\section{Introduction}

Many etiologies are involving abdominal pain pathophysiology. Abdominal pain may be caused by functional problems like gastroenteritis or organic problems such as malignancy, bowel obstruction and mesenteric ischemia [1]. Decreased demand in arterial blood supply of intestine results in mesenteric ischemia [2].

Celiac artery compression syndrome (CACS) is one of the rare etiologies of mesenteric ischemia. The main reason is the external compression of celiac artery by the median arcuate ligament (MAL). The median arcuate ligament is a fibrous band that connects the left and right diaphragmatic crura across the aortic hiatus at the level of the T12/L1 vertebral bodies, although the normal anatomical position is variable. The MAL crosses the aorta anteriorly but its location relative to the origin of the celiac axis varies [3]. Imaging techniques including Doppler ultrasound (US), computed tomography (CT), magnetic resonance imaging (MRI) and selective catheter angiography can be used for diagnosis. 
The symptoms of the celiac artery compression syndrome (CACS) involve nausea, vomiting, weight loss and abdominal pain. Exact mechanism of abdominal pain is uncertain. It can be based on either ischemia secondary to celiac artery compression or neuropathic pain due to celiac splenic nerve involvement [4]. The compression of the celiac trunk is usually seen on the saggittal view of a CT scan where it appears prominent on expiration; however, in severe cases, it may be shown as a persistent compression on inspiration [5]. Treatment strategies include surgical division of median arcuate ligament to relieve external compression either by conventional open surgery or laparoscopic approach [6]. Percutaneous endovascular treatment is an alternative technique which may be considered in selected cases [7].

Herein we report a celiac artery compression syndrome case presenting with frequently seen gastrointestinal symptoms such as abdominal pain and weight loss, which was successfully treated by open surgical division to emphasize early consideration of CACS in the etiology of abdominal pain.

\section{Case Report}

A 79-year-old woman admitted to our emergency department with a 2-month history of abdominal pain and 6 kg weight loss in 2 months. She was a housewife and denied systemic disease in the past. She did not smoke or drink alcohol before. Abdominal pain was located at the epigastric area, starting 40 minutes after meals and last in for 2.5 hours. The pain was relieved by bending forward. She also mentioned diarrhea two to four times per day. No vomiting or fever was noted.

On physical examination, her abdomen was soft with mild tenderness at the epigastric area; bowel sound was hyperactive without bruit. The laboratory examination was unremarkable. No leukocytosis was observed. Direct abdominal x-ray revealed nonspecific radiolucent areas. The abdominal ultrasonography had no abnormalities.

During hospitalization, malignancies were considered initially and the esophagogastroduodenoscopy and colonoscopy demonstrated no particular abnormality. However, her cramping abdominal pain persisted. Thus, under the suspicion of ischemic bowel disease, color doppler sonography was performed and attenuated blood flow on celiac artery was observed. The patient subsequently underwent CT-angiography which showed a short segment stricture with post-stenotic dilatation at the root of celiac trunk and found the median arcuate ligament compressing the root of celiac trunk (Figure 1). Thus, the radiological findings and the patients' symptoms were consistent with diagnosis of CACS.

According to the studies in the literature, surgical treatment was suggested. Our surgery team preferred upper midline incision and it was found that celiac artery have been compressed by median arcuate ligament. Fibrous structure was divided to relieve the extrinsic compression. Postoperative period was uneventful. She was successfully treated by surgery. On clinical follow up normal weight gain was achieved and her clinical symptoms improved.

\section{Discussion}

The CACS or Median arcuate ligament syndrome (MALS) is a rare cause of mesenteric ischemia and weight loss. Harjola described this syndrome in 1963. It is estimated that in $10 \%-24 \%$ of normal, asymptomatic individuals the median arcuate ligament crosses anterior to the celiac artery, causing some degree of compression. Approximately $1 \%$ of these individuals exhibit severe compression associated with symptoms of MALS. The syndrome most commonly affects individuals between 20 and 40 years old, particularly thin women. Celiac artery blood flow may be also restricted by celiac ganglion and type B aortic dissection. Median arcuate ligament syndrome is a diagnosis of exclusion of other abdominal pain reasons [5]. The diagnosis of MALS is generally considered only after patients have undergone an extensive evaluation of the gastrointestinal tract including eusophagogastroduodenoscopy and colonoscopy [8].

CACS includes the characteristic clinical triad of postprandial pain, diarrhea and weight loss. An abdominal bruit that intensifies with expiration may be heard in the epigastric region is an additional finding [9].

Diagnostic imaging for MALS is divided into screening and confirmatory tests. A reasonable screening test for patients with suspected MALS is duplex ultrasonography to measure blood flow through the celiac artery peak systolic velocities greater than $200 \mathrm{~cm} / \mathrm{s}$ are suggestive of celiac artery stenosis associated with MALS. Blood flow through the celiac artery can also be evaluated intraoperatively with the duplex ultrasonography [8].

The newly developed multi-detector CT angiography has become a more favorable modality in diagnosing 


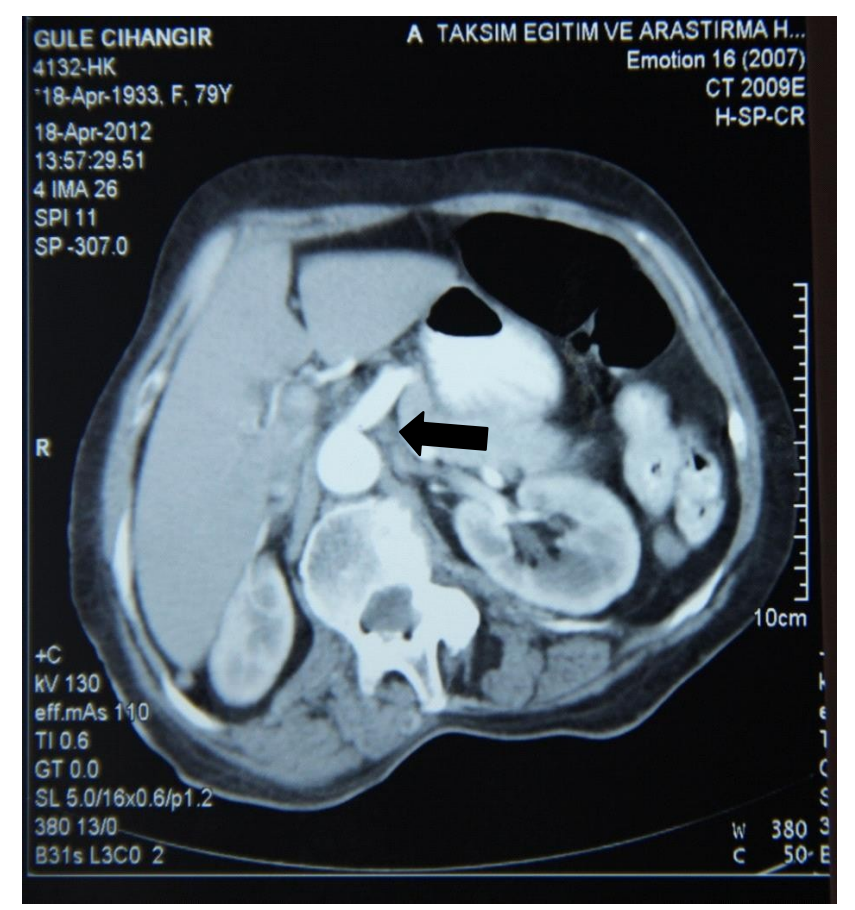

Figure 1. Axial CT image in the arterial phase show compression of the celiac axis by the median arcuate ligament with poststenotic dilatation.

mesenteric ischemia because it is rapid, non-invasive and non expensive. It is useful in evaluating the presence and degree of stenosis of the celiac trunk and SMA, demonstrating the collateral circulation, also helpful in excluding other cause of vascular obstruction. Vessels, which are small and have wall abnormalities with a complex anatomic configuration, can easily be visualized with proper processing techniques. However, the limitations of this modality include the lack of dynamic representation of flow abnormalities and difficulty in evaluating heavily calcified vessels [10] [11].

In the present patient we performed duplex ultrasonography to measure blood flow through the celiac artery and the external compression was proved. Then CT angiography identified a narrowing at the level of celiac artery origin with post-stenotic dilatation (Figure 1). Cases without post stenotic dilatation and with collateral vascular vessels are also reported [12].

Treatment modality of the CACS is decompression of the celiac artery. The mainstay of treatment involves an open surgical approach to divide the MAL. The removal of celiac ganglia may be combined with this procedure. [8]. Recently laparoscopic approach is frequently used, however carries higher risk of artery injury and massive hemorrhage then open surgical division. Despite that clinical outcomes are similar [13]. Percutaneous transluminal angioplasty (PTA) may also be used to achieve celiac artery decompression in patients cannot undergo an open surgical procedure [4] [8].

In the present patient surgical team performed conventional laparotomy for division of the median arcuate ligament. The patient's symptoms improved dramatically after the laparotomy. In a large study named long-term evaluation of the celiac band syndrome, it has reported $83 \%$ of patients were asymptomatic in the first 6 months after decompression, but only $41 \%$ of patients remained asymptomatic 3 to 11 years later [4] [14] [15]. The present patient had a 3-year symptom-free follow up period which confirmed postoperative success.

\section{Conclusion}

CACS is a rare cause of mesenteric ischemia and difficult postprandial pain clinically. However, it must be considered in patients presenting postprandial pain without other causes of abdominal pain. CT angiography and duplex ultrasonography are main diagnostic tools. On follow-up symptom, improvement shows postoperative success. 


\section{Ethical Approval}

Patient had given written consent for the treatment and for the case report to be published.

\section{References}

[1] Clouse, R.E., Mayer, E.A., Aziz, A., Drossman, D.A., Dumitrascu, D.L., Mönnikes, H. and Naliboff, B.D. (2006) Functional Abdominal Pain Syndrome. Gastroenterology, 130, 1492-1497. http://dx.doi.org/10.1053/j.gastro.2005.11.062

[2] Martinez, J.P. and Hogan, G.J. (2004) Mesenteric Ischemia. Emergency Medicine Clinics of North America, 22, 909928. http://dx.doi.org/10.1016/j.emc.2004.05.002

[3] Paz, Z., Rak, Y. and Rsen, A. (1991) Anatomical Basis for Celiac Trunk and Superior Mesenteric Artery Entrapment. Clinical Anatomy, 4, 256-264. http://dx.doi.org/10.1002/ca.980040404

[4] Carbonell, A.M., Kercher, K.W., Heniford, B.T. and Matthew, B.D. (2005) Laparoscopic Management of Median Arcuate Ligament Syndrome. Surgical Endoscopy and Other Interventional Techniques, 19, 729. http://dx.doi.org/10.1007/s00464-004-6010-x

[5] Horton, K.M., Talamini, M.A. and Fishman, E.K. (2005) Median Arcuate Ligament Syndrome: Evaluation with CT Angiography. Radiograph, 25, 1177-1182. http://dx.doi.org/10.1148/rg.255055001

[6] Duffy, A.J., Panait, L., Eisenberg, D., Bell, R.L., Roberts, K.E. and Sumpio, B. (2009) Management of Median Arcuate Ligament Syndrome: A New Paradigm. Annals of Vascular Surgery, 23, 778-784. http://dx.doi.org/10.1016/j.avsg.2008.11.005

[7] Hongsakul, K., Rookkapan, S., Sungsiri, J. and Tubtawee, T. (2012) A Severe Case of Median Arcuate Ligament Syndrome with Successful Angioplasty and Stenting. Case Reports in Vascular Medicine, 2012, Article ID: 129870.

[8] Duncan, A.A. (2008) Median Arcuate Ligament Syndrome. Current Treatment Options in Cardiovascular Medicine, 10, 112-116. http://dx.doi.org/10.1007/s11936-008-0012-2

[9] Roayaie, S., Jossart, G., Gitlitz, D., Lamparello, P., Hollier, L. and Gagner, M. (2000) Laparoscopic Release of Celiac Artery Compression Syndrome Facilitated by Laparoscopic Ultrasound Scanning to Confirm Restoration of Flow. Journal of Vascular Surgery, 32, 814-817. http://dx.doi.org/10.1067/mva.2000.107574

[10] Joshi, A.R., Nalavde, S., Merchant, S. and Gujone, S. (2004) Renal Vasculature Evaluation Using a Multidetector CT Scanner: A Brief Study of 20 Cases. Indian Journal of Radiology \& Imaging, 14, 373-378.

[11] Joshi, A., Nimbkar, V., Merchant, S., Mhashelkar, Y. and Talekar, K. (2004) The Role of CT Angiography in the Evaluation of Peripheral Vasculature Using MSCT-Our Initial Experience. Indian Journal of Radiology \& Imaging, 14, 309-315.

[12] Tembey, R.A., Bajaj, A.S., Wagle, P.K. and Ansari, A.S. (2015) Real-Time Ultrasound: Key Factor in Identifying Celiac Artery Compression Syndrome. Indian Journal of Radiology \& Imaging, 25, 202-205. http://dx.doi.org/10.4103/0971-3026.155882

[13] Kim, S.J., Park, Y.J., Yang, S.S. and Kim, Y.W. (2013) Open Surgical Decompression of Celiac Axis Compression by Division of the Median Arcuate Ligament. Journal of the Korean Surgical Society, 85, 93-95. http://dx.doi.org/10.4174/jkss.2013.85.2.93

[14] Gander, S., Mulder, D., Jones, S., Ricketts, J.D., Soboleski, D.A. and Justinich, C.J. (2010) Recurrent Abdominal Pain and Weight Loss in an Adolescent: Celiac Artery Compression Syndrome. Canadian Journal of Gastroenterology \& Hepatology, 24, 91-93.

[15] Evans, W.E. (1974) Long Term Evaluation of the Celiac Band Syndrome. Surgery, 76, 867-871. 